\title{
Avaliação Comparativa de Sistemas de Saúde com a Utilização de Fronteiras Estocásticas: Brasil e OCDE*
}

\author{
Alexandre Marinho ${ }^{\dagger}$, Simone de Souza Cardoso ${ }^{\ddagger}$, Vivian Vicente de \\ Almeida ${ }^{\S}$
}

Contents: 1. Introdução; 2. A Amostra e os Dados Utilizados; 3. As Fronteiras Estocásticas; 4. Apresentação e Discussão dos Resultados; 5. Comentários Finais; A. Apêndice I: A Correlação entre Variáveis; B. Apêndice II: A Escolha das Variáveis.

Palavras-chave: Brasil e OCDE; Sistemas de Saúde; Fronteiras Estocásticas (Stochastic Frontiers SF).

Códigos JEL: $\quad$ C61; D61; H51; I12; I18.

Avaliamos a eficiência na provisão de serviços de saúde no Brasil, comparado com os países da Organização para Cooperação e Desenvolvimento Econômico (OCDE). Estimamos, em que medida, variáveis como: gasto per capita em saúde; esperança de vida ao nascer; e índice de sobrevivência infantil; servem de representação para um serviço de saúde eficiente, dado o gasto per capita com saúde. Foram geradas fronteiras de eficiência, calculadas em modelos de fronteiras estocásticas (stochastic frontiers), e os resultados, em termos relativos, não são totalmente desfavoráveis ao Brasil. Nosso país, a despeito dos indicadores de saúde desfavoráveis em relação aos países da amostra tem, em termos de eficiência técnica relativa, o melhor desempenho relativo em todos os anos da análise.

We evaluate the efficiency of expenditure in health care provision by comparing output measures (life expectancy; infant survival rate) from the health system of Brazilian and OECD countries with an expenditure measure (health expenditure per capita). We estimated the efficiency frontier and

\footnotetext{
* Pesquisa realizada com o apoio do Programa de Pesquisa para o Desenvolvimento Nacional - PNPD. Agradecemos os valiosos comentários recebidos, que aprimoraram o texto. Todos os eventuais erros remanescentes são de nossa inteira responsabilidade.

† Técnico de Planejamento e Pesquisa do Instituto de Pesquisa Econômica Aplicada (IPEA) e Professor Adjunto da Faculdade de Ciências Econômicas da Universidade Estadual do Rio de Janeiro (UERJ). Endereço para correspondência: Instituto de Pesquisa Econômica Aplicada - IPEA. Av. Presidente Antonio Carlos 51/10. Centro, RJ. CEP:20.020-010. Tel: (21) 3515-8621/3515-8626. E-mail: alexandre.marinho@ipea.gov.br

${ }^{\ddagger}$ Assistente de Pesquisa no IPEA. E-mail: simone.cardoso@ipea.gov.br

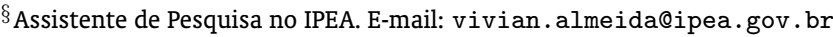


country rankings by using stochastic frontiers. Although the Brazilian health indicators are not acceptable, our results provide some evidence that Brazil is not in the worst relative position in the efficiency ranking.

\section{INTRODUÇÃO}

De acordo com a Organização Mundial de Saúde - OMS, entre as destinações que definem um sistema de saúde encontram-se melhorar e proteger a saúde das pessoas; promover a equidade no financiamento da atenção à saúde; atender às expectativas da população no que se refere às condições de atendimento (responsiveness); e reduzir as desigualdades (OMS, 2000). Portanto, a avaliação desses sistemas é uma obrigação dos governos, gestores, pesquisadores, e usuários. No Brasil, a eficiência na administração pública direta e indireta de qualquer dos Poderes da União, dos Estados, do Distrito Federal e dos Municípios é imperativo constitucional (Brasil, 2006).

No presente trabalho, realizamos avaliações de eficiência que procuram inferir, em que medida o gasto per capita, em termos monetários, no sistema de saúde do Brasil, comparado com os sistemas de saúde dos países da $\mathrm{OCDE},{ }^{1}$ seria eficiente em maximizar indicadores (ou minimizar custos) relacionados ao desempenho desses sistemas. Estimamos a magnitude em que variáveis tais como: esperança de vida ao nascer para homens; esperança de vida ao nascer para mulheres; mortalidade infantil (ou o complemento dessa medida: o índice de sobrevivência infantil); podem servir de reflexo para um serviço de saúde eficiente vis à vis a magnitude do gasto per capita com saúde nas sociedades analisadas.

Em Afonso e Aubyn (2005), que realizaram um estudo referente aos sistemas de saúde dos países da OCDE, o México e a Turquia são excluídos da amostra, pois são considerados outliers e o Brasil, por não fazer parte da OCDE, não é incluído na análise. No presente texto, e em Ocké-Reis (2006), encontram-se indicativos de que existem significativas diferenças entre os indicadores de saúde do Brasil e os países desenvolvidos da OCDE. Essas dissimilaridades, que seriam sempre desfavoráveis ao Brasil, e a outros países em desenvolvimento, são observadas em indicadores tais como: taxa de mortalidade infantil; expectativa de vida; gasto per capita em saúde; participação do gasto público em saúde no total do gasto; a oferta de profissionais de saúde (médicos e enfermeiros) e de leitos hospitalares.

No presente estudo, ao contrário do que ocorreu em Afonso e St Aubyn, op. cit., o Brasil é incluído, e México e Turquia são mantidos na amostra. Conforme veremos, dados os seus indicadores mais gerais de saúde, México e Turquia servem como importantes referências para análise do desempenho do nosso país. Ademais, no presente texto, utilizaremos técnicas elaboradas justamente para a deteç̧ão, e para a acomodação, de dissimilaridades em amostras que sejam objeto de avaliações de eficiência.

O Brasil, apesar dos indicadores de saúde desfavoráveis em relação aos países da amostra, tem, em termos de eficiência técnica relativa, o melhor desempenho em todos os anos da análise.

O estudo é baseado na metodologia conhecida como Fronteiras Estocásticas - SF (Stochastic Frontiers). As SF foram desenvolvidas, de modo simultâneo, em Aigner et alii (1977), Battese e Corra (1977), e Meeusen e Van Den Broeck (1977). Os modelos utilizados de SF, por razões de conveniência metodológica, no presente trabalho, ${ }^{2}$ seguem o método de minimização de custo. Cabe destacar que, além da inclusão do componente aleatório neste método, o modelo estocástico apresenta a classificação dos países segundo o grau de ineficiência.

O estudo é composto de cinco Seções. Além desta Introdução, na Seção 2, apresentamos a amostra, as variáveis, e os dados utilizados. Na Seção 3, introduzimos a metodologia utilizada, as Fronteiras

\footnotetext{
${ }^{1}$ Organização para a Cooperação e Desenvolvimento Econômico - OCDE (Organization for Economic Co-Operation and Development - OECD): Alemanha, Austrália, Áustria, Bélgica, Canadá, Coréia, Dinamarca, Eslováquia, Espanha, Estados Unidos, Finlândia, França, Grécia, Holanda, Hungria, Islândia, Irlanda, Itália, Japão, Luxemburgo, México, Nova Zelândia, Noruega, Polônia, Portugal, Reino Unido, República Tcheca, Suécia, Suíça, Turquia).

${ }^{2} \mathrm{~A}$ utilização de modelos de múltiplos outputs em FE sofrem percalços consideráveis.
} 
Estocásticas - SF (Stochastic Frontiers). Na Seção 4 as SF são aplicadas em nossa amostra, e os resultados obtidos são analisados. Na Seção 5 estão os nossos Comentários Finais.

\section{A AMOSTRA E OS DADOS UTILIZADOS}

Os países analisados: Alemanha, Austrália, Áustria, Bélgica, Brasil, Canadá, Coréia, Dinamarca, Eslováquia, Espanha, Estados Unidos, Finlândia, França, Grécia, Holanda, Hungria, Islândia, Irlanda, Itália, Japão, Luxemburgo, México, Nova Zelândia, Noruega, Polônia, Portugal, Reino Unido, República Tcheca, Suécia, Suíça, Turquia.

O estudo abrange um painel para os anos de 2004, 2005 e 2006, por razões de disponibilidade de dados.

As variáveis utilizadas: Um quadro-resumo de indicadores clássicos de saúde nos países da amostra está na Tabela 1.

Table 1: Estatística descritiva de indicadores selecionados (OCDE com Brasil e Brasil)

\begin{tabular}{|c|c|c|c|c|c|c|c|c|c|}
\hline \multirow[b]{2}{*}{ Variáveis } & & \multirow[b]{2}{*}{ Observações } & \multirow[b]{2}{*}{ Média } & \multirow[b]{2}{*}{$\begin{array}{l}\text { Média } \\
\text { (Brasil) }\end{array}$} & \multirow[b]{2}{*}{$\begin{array}{l}\text { Desvio- } \\
\text { padrão }\end{array}$} & \multirow[b]{2}{*}{ Min. } & \multirow[b]{2}{*}{ Max. } & \multicolumn{2}{|c|}{ 95\%conf.interval. } \\
\hline & & & & & & & & Inf. & Sup. \\
\hline $\begin{array}{l}\text { Gasto total em saúde } \\
\text { per capita }\end{array}$ & Overall & $\mathrm{N}=93$ & $2,682.28$ & 742.33 & $1,331.11$ & 572.00 & $6,714.00$ & $2,408.14$ & $2,956.42$ \\
\hline \multirow[t]{2}{*}{ (US\$ PPP) } & Between & $\mathrm{n}=31$ & & & $1,339.97$ & 603.00 & $6,358.33$ & & \\
\hline & Within & $\mathrm{T}=3$ & & & 123.97 & $2,337.95$ & $3,037.95$ & & \\
\hline $\begin{array}{l}\text { Esperança de vida ao } \\
\text { nascer }\end{array}$ & Overall & $\mathrm{N}=93$ & 75,452 & 67,667 & 3,154 & 67,000 & 79,000 & 74,802 & 76,101 \\
\hline \multirow[t]{2}{*}{ Homens } & Between & $\mathrm{n}=31$ & & & 3,152 & 67,667 & 79,000 & & \\
\hline & Within & $\mathrm{T}=3$ & & & 0,474 & 74,118 & 76,785 & & \\
\hline $\begin{array}{l}\text { Esperança de vida ao } \\
\text { nascer }\end{array}$ & Overall & $N=93$ & 81,183 & 74,667 & 2,702 & 73,000 & 86,000 & 80,626 & 81,739 \\
\hline \multirow[t]{2}{*}{ Mulheres } & Between & $\mathrm{n}=31$ & & & 2,697 & 74,000 & 86,000 & & \\
\hline & Within & $\mathrm{T}=3$ & & & 0,434 & 79,849 & 82,183 & & \\
\hline \multirow{3}{*}{$\begin{array}{l}\text { Mortalidade infantil } \\
\text { (mil nascidos vivos) }\end{array}$} & Overall & $\mathrm{N}=93$ & 6,376 & 26,333 & 6,564 & 2,000 & 32,000 & 5,025 & 7,728 \\
\hline & Between & $\mathrm{n}=31$ & & & 6,523 & 2,000 & 26,333 & & \\
\hline & Within & $\mathrm{T}=3$ & & & 1,207 & $-0,957$ & 12,043 & & \\
\hline População & Overall & $\mathrm{N}=93$ & $43.938,310$ & $187.377,700$ & $63.597,810$ & 295,000 & $302.841,000$ & $30.840,500$ & $57.036,120$ \\
\hline \multirow{2}{*}{$\begin{array}{l}\text { (milhares } \\
\text { de habitantes) }\end{array}$} & Between & $\mathrm{n}=31$ & & & $64.298,470$ & 296,000 & $299.755,700$ & & \\
\hline & Within & $\mathrm{T}=3$ & & & 513,774 & $42.395,650$ & $47.023,650$ & & \\
\hline
\end{tabular}

Comparado com a amostra, em um intervalo de confiança de $95 \%$, conforme a Tabela 1, o Brasil tem desempenho ruim nos indicadores de saúde, como esperança de vida ao nascer (para homens e para mulheres) e mortalidade infantil. O Brasil tem gasto per capita em saúde relativamente baixo; e é bastante populoso.

A lista de variáveis efetivamente utilizadas, listadas a seguir, foram escolhidas de modo a compatibilizar o nosso trabalho com a literatura de caráter epidemiológico e de saúde pública (e. g. Dever, 1998, OMS, 2000, Rouquayrol e Almeida Filho, 2001) e de economia da saúde (e. g. Barros, 2005, Santerre e Neun, 2000, Zweifel e Breyer, 1997).

As bases de dados utilizadas foram publicadas pela Organização Mundial de Saúde - OMS (http:// www . who.int/whosis/whostat2007.pdf). A lista de variáveis inicial é a seguinte: Recursos (inputs): Gasto com Saúde per capita (em US\$ Paridade do Poder de Compra). Resultados (outputs): Esperança 
de Vida ao Nascer para Homens; Esperança de Vida ao Nascer para Mulheres; Índice de Mortalidade Infantil; Índice de Sobrevivência Infantil.

Observações com relação às variáveis utilizadas: Para permitir o uso nos modelos de custos que utilizamos, modificamos as variáveis de resultados que os sistemas de saúde tentam reduzir, como Mortalidade Infantil e Anos de Vida Perdidos, conforme explicamos a seguir.

No caso da variável Mortalidade Infantil foi calculada uma variável complementar ao indicador; o Índice de Sobrevivência Infantil, que os sistemas de saúde buscariam, ceteris paribus, aumentar. Mais especificamente, procurou-se estimar, de um total de 1000 crianças nascidas, quantas conseguem sobreviver 12 meses após o nascimento. O cálculo, conforme Afonso e Aubyn (2005), foi feito da seguinte forma:

$$
I S R=\frac{1000-I M R}{I M R}
$$

onde, IMR: Índice de Mortalidade Infantil (Infant Mortality Rate).

Conforme ressaltam Afonso e Miguel St. Aubyn, op. cit., esse indicador aumenta com a melhoria das condições de saúde. Além disso, ele reflete uma razão entre a taxa de crianças que sobreviveram até um ano de idade e a taxa de crianças que morreram antes de completar essa idade. ${ }^{3}$ Consideremos, no caso brasileiro, um valor aproximado para o Índice de Mortalidade Infantil - IMR, igual a 32 mortes por cada mil crianças nascidas vivas.

Assim, $I M R=32$. O Índice de Sobrevivência Infantil é calculado da seguinte forma:

10. Passo: Seja a quantidade de sobreviventes a cada 1000 crianças nascidas vivas $I S R^{*}=1000-I M R$. Então, $I S R^{*}=1000-32=968$.

2o. Passo: Calcular o IRS. Esse é computado da seguinte forma:

$$
I S R=\frac{1000-I M R}{I M R}=\frac{I S R^{*}}{I M R}=30,25
$$

Caberia, ainda, um questionamento: por que utilizar o ISR e não o indicador mais simples e direto, o $I R S^{*}=1000-I M R$ ? A resposta está na sensibilidade do ISR a mudanças no Índice Mortalidade Infantil - IMR. O ISR é mais sensível, e responde de maneira mais fidedigna do que o ISR* às mudanças no IMR, no intervalo relevante. Exemplos fictícios seguem abaixo:

1) Suponha que o Índice de Mortalidade Infantil dobre, ou seja, passe de $I M R=32$ para $I M R=64$. Nesse caso,

$$
\begin{aligned}
I S R^{*} & =1000-64=936 \\
I S R & =\frac{1000-64}{64}=14,625
\end{aligned}
$$

2) Agora, suponhamos que o Índice de Mortalidade Infantil se reduza à metade, ou seja, passe de $I M R=32$ para $I M R=16$. Nesse caso,

$$
\begin{aligned}
I S R^{*} & =1000-16=984 \\
I S R & =\frac{1000-16}{16}=61,5
\end{aligned}
$$

Vemos que o ISR* varia muito pouco quando o IMR dobra, ou quando ele é dividido por dois. Já o ISR cai para um valor que é praticamente a metade quando o IMR é dividido por dois e, praticamente, dobra quando o IMR é multiplicado por dois.

\footnotetext{
${ }^{3}$ Seja a quantidade de sobreviventes a cada 1000 crianças nascidas vivas $I S R^{*}=1000-I M R$. O indicador $I S R=$ $\frac{1000-I M R}{I M R}=\frac{I S R^{*}}{I M R}$.
} 


\section{AS FRONTEIRAS ESTOCÁSTICAS}

As fronteiras estocásticas - SF (Stochastic Frontier), devidas a Aigner et alii (1977); Battese e Corra (1977); e Meeusen e Van Den Broeck (1977); são modelos de regressão com uma perturbação assimétrica não normal, motivados pela idéia de que desvios da fronteira de produção podem não estar inteiramente sob o controle das unidades produtivas, ou unidades tomadoras de decisão (decision making units DMUs). No caso de modelos determinísticos, todos os desvios da produção causados, por erros de medida, ou por má especificação do modelo seriam avaliados como ineficiência. A interpretação, nos modelos de SF, é que cada unidade produtiva se defronta com a sua própria fronteira de produção, e que essa fronteira é aleatoriamente determinada pelo conjunto de todos os elementos estocásticos que entrariam no modelo, fora do controle da DMU. Assim, a fronteira não passa, necessariamente, por todos os pontos de produção mais elevada, ou de mais baixo custo.

As Fronteiras Estocásticas têm ampla utilização na avaliação de serviços de saúde (para exemplos na literatura, consultar, entre outros, Vitaliano e Toren, 1994, Evans et alii, 2000, OMS, 2000, Jacobs et alii, 2006, Estache et alii, 2007, Souza et alii, 2010).

A formulação geral para uma fronteira de produção, como nos modelos de regressão:

$$
\begin{aligned}
y & =\beta x^{\prime}+\varepsilon \\
\operatorname{com} \varepsilon & =v-u
\end{aligned}
$$

onde;

$y$ é o produto, $x$ os insumos;

$\varepsilon$ é o componente estocástico;

$u$ é não-negativo e $v$ tem distribuição de probabilidades livre.

Assume-se que $v$ e $u$ são independentes.

O componente $v$ não está sob o controle da DMU, e $u$ é um termo não negativo, que captura a ineficiência e define a que distância a DMU está da fronteira produtiva. Não existe critério econômico para definir a escolha da distribuição de probabilidades de $u$. É usual supor que $v$ é normalmente distribuída, ou seja, $v \sim N\left[0, \sigma_{v}^{2}\right]$.

Em uma fronteira para custos, teremos

$$
\begin{aligned}
c & =c(y, w)+\varepsilon \\
\operatorname{com} \varepsilon & =v+u
\end{aligned}
$$

Nesse caso, $c$ são os custos e $w$ é o custo unitário (preços) de cada um dos fatores de produção. Valem, para a fronteira de custos, as demais definições e restrições da fronteira de produção. Para mais detalhes técnicos sobre as Fronteiras Estocásticas ver, Fried et alii (1993) e Jacobs et alii (2006).

\subsection{A seleção das variáveis e o modelo utilizado}

Na amostra existe correlação entre a esperança de vida ao nascer para homens e a esperança de vida ao nascer para mulheres. Então, utilizamos o critério de informação de Akaike inclusivo - AIC que selecionou, entre essas duas variáveis, a esperança de vida ao nascer para homens como a melhor variável para o modelo (ver Apêndice). O AIC é uma estimativa da logverossimilhança negativa dos modelos, ponderada para o número de parâmetros estimados. Consequentemente, o modelo com menor valor de AIC é o mais apropriado sendo que: 


$$
A I C=-2 \log (L) / n+(2 K / n)
$$

onde:

$L=$ máxima verossimilhança;

$K=$ número de parâmetros do modelo;

$n=$ número de observações.

Quando o ajuste é bom o modelo explica boa parte da variação total e, consequentemente, o valor de $\mathrm{R}^{2}$ é próximo de 1 . Para mais detalhes sobre o método AIC, ver Maddala (2001), especialmente o Capítulo 12 .

Após a aplicação do AIC escolhemos com variáveis explicativas do modelo a Esperança de Vida ao Nascer para Homens; e o Índice de Sobrevivência Infantil.

A utilização de uma função do tipo time-varying translog (Schmidt, 1988), foi considerada. Assim, idealmente, além dos custos e dos outputs, deveríamos incluir os preços dos insumos no modelo. Entretanto, tais preços não estão disponíveis, pois preços consistentes são de difícil obtenção em uma amostra de diversos países, que utilizam vários insumos que não são comercializados em mercado. Adicionalmente, vemos que a quantidade de observações é pequena para tal especificação (93 observações). Também tínhamos os já citados problemas de correlação entre os outputs, e a elasticidade de substituição entre os outputs utilizados não era de interesse primordial. Então, após várias tentativas e problemas de convergência, adotamos a especificação parcimoniosa do tipo time-invariant Cobb-Douglas. Como é usual em modelos econométricos, para reduzir problemas de heteroscedasticidade, para permitir a leitura direta das elasticidades, ${ }^{4}$ e para facilitar a convergência dos modelos, foram aplicados logaritmos naturais em todas as variáveis.

O modelo final utilizado foi um Cobb-Douglas com ineficiência não variável no tempo (time-invariant Cobb-Douglas model):

$$
\ln \left(C_{i t}\right)=\beta_{0}+\beta 1 \ln \left(X_{1 i t}\right)+\beta_{2} \ln \left(X_{2 i t}\right)+v_{i t}+u_{i}
$$

onde:

$u_{i}$ é uma perturbação não-negativa, normal truncada, e iid. $u_{i} \sim N^{+}\left(\mu, \sigma_{\mu}^{2}\right)$;

$v_{i t}$ é normal e $i i d . v_{i t} \sim N\left(0, \sigma_{v}^{2}\right)$;

$C_{i t}=$ logaritmo natural do gasto com saúde per capita (US\$ PPP);

$X_{1 i t}=$ logaritmo natural da Esperança de Vida ao Nascer para Homens;

$X_{2 i t}=$ logaritmo natural do Índice de Sobrevivência Infantil.

O software utilizado foi o STATAtm 9.0, Statistics/Data Analysis da Stata Corp que maximiza a função de máxima verossimilhança logarítmica com o uso do método de Newton-Raphson. A estimativa da matriz de variância-covariância é calculada como o inverso da matriz Hessiana (matriz das segundas derivadas parciais).

\footnotetext{
${ }^{4} \mathrm{~A}$ elasticidade mede a relação entre a variação percentual dos regressores e a variação percentual da variável dependente.
} 


\section{APRESENTAÇ̃̃O E DISCUSSÃO DOS RESULTADOS}

\subsection{Apresentação dos resultados}

As Tabelas 2 e 3; o Quadro 1; e as Figuras 1 e 2 apresentam os principais resultados obtidos no modelo utilizado (Equação 6). ${ }^{5}$ Ressaltamos, novamente, que os modelos de SF evidenciam ineficiências. Os resultados das regressões são discutidos a seguir.

Table 2: Time-invariant inefficiency model

\begin{tabular}{|c|c|c|c|c|c|c|}
\hline \multirow[t]{2}{*}{ Gasto com saúde per capita (US\$ PPP) } & \multirow[t]{2}{*}{ Coeficiente } & \multirow[t]{2}{*}{ Desvio padrão } & \multirow[t]{2}{*}{$Z$} & \multirow[t]{2}{*}{$P>|z|$} & \multicolumn{2}{|c|}{ Intervalo de confiança } \\
\hline & & & & & Low & Up \\
\hline Intercepto & $-16,021$ & 2,812 & $-5,700$ & 0,000 & $-21,533$ & $-10,510$ \\
\hline Esperança de Vida ao nascer (homens) & 5,168 & 0,669 & 7,730 & 0,000 & 3,858 & 6,478 \\
\hline Índice de Sobrevivência infantil & 0,122 & 0,055 & 2,210 & 0,030 & 0,014 & 0,230 \\
\hline$/ \mathrm{mu}$ & 0,742 & 0,157 & 4,720 & 0,000 & 0,434 & 1,051 \\
\hline /nsigma2 & $-1,673$ & 0,344 & $-4,860$ & 0,000 & $-2,347$ & $-0,998$ \\
\hline /ilgtgamma & 4,694 & 0,400 & 11,730 & 0,000 & 3,910 & 5,478 \\
\hline sigma2 & 0,188 & 0,065 & & & 0,096 & 0,369 \\
\hline gamma & 0,991 & 0,004 & & & 0,980 & 0,996 \\
\hline sigma_u2 & 0,186 & 0,065 & & & 0,059 & 0,313 \\
\hline sigma_v2 & 0,002 & 0,000 & & & 0,001 & 0,002 \\
\hline Modelo & Obs. & ll(null) & ll(modelo) & $\mathrm{df}$ & AIC & BIC \\
\hline . & 93 & . & 78,350 & 6 & $-144,701$ & $-129,505$ \\
\hline \multicolumn{7}{|c|}{ Group variable (i): Países; Time variable (t): Tempo; } \\
\hline \multicolumn{7}{|c|}{ Number of obs $=93 ;$ Number of groups $=31 ;$ Obs per group: 3} \\
\hline \multicolumn{7}{|c|}{ Wald chi2 $(2)=79,780 ;$ Log likelihood $=78,350 ;$ Prob $>$ chi2 $=0,000$} \\
\hline
\end{tabular}

\subsection{Discussão dos resultados}

A Esperança de Vida ao Nascer (homens) e o Índice de Sobrevivência infantil se correlacionam positivamente com o Gasto per Capita em Saúde. Na amostra, em média, países que gastam mais, têm melhores indicadores de saúde.

Nos períodos analisados, a maioria dos países se mostrou levemente menos ineficientes no ano de 2004 do que nos períodos de 2005 e 2006. O Brasil mostra uma tendência crescente em se tornar mais ineficiente, mas também os outros países, em média, tendem a uma ineficiência crescente. Vale ressaltar também, que no caso dos Estados Unidos observa-se uma leve tendência de queda da ineficiência.

Ressalte-se que, conforme a Tabela 1, o Brasil, mesmo com baixo nível de gasto per capita médio (Brasil: US\$PPP 742.33 e amostra: US\$PPP 2,628.28) vem apresentando uma melhora acelerada nos

\footnotetext{
${ }^{5}$ A eficiência técnica $(E T)$, pode ser obtida por: $E T \exp (u)$. Essa formulação permite obter os targets $\left(C^{*}\right)$ de custo sobre a fronteira estimada fazendo: $C^{*}=C \times E T$ bem como a mudança percentual $(P)$ requerida para tanto, obtida como $P=E T-1$.
} 
Table 3: Fronteira de ineficiência (Painel)

\begin{tabular}{|c|c|c|c|c|c|}
\hline \multirow[t]{2}{*}{ Países } & \multicolumn{4}{|c|}{ Ineficiências } & \multirow{2}{*}{$\begin{array}{l}\text { Ranking médio } \\
\text { Posição }\end{array}$} \\
\hline & 2004 & 2005 & 2006 & Média & \\
\hline Alemanha & 7,031 & 7,031 & 7,082 & 7,192 & 15 \\
\hline Austrália & 7,158 & 7,209 & 7,209 & 7,056 & 26 \\
\hline Áustria & 7,003 & 7,082 & 7,082 & 7,031 & 18 \\
\hline Bélgica & 6,979 & 7,031 & 7,082 & 6,197 & 12 \\
\hline Brasil & 6,101 & 6,222 & 6,269 & 7,158 & 1 \\
\hline Canadá & 7,158 & 7,158 & 7,158 & 6,892 & 25 \\
\hline Coréia & 6,796 & 6,929 & 6,951 & 7,025 & 8 \\
\hline Dinamarca & 6,979 & 7,031 & 7,066 & 6,548 & 11 \\
\hline Eslováquia & 6,548 & 6,548 & 6,548 & 7,117 & 4 \\
\hline Espanha & 7,082 & 7,082 & 7,186 & 6,916 & 22 \\
\hline Estados Unidos & 6,929 & 6,909 & 6,909 & 7,049 & 9 \\
\hline Finlândia & 7,014 & 7,066 & 7,066 & 7,065 & 16 \\
\hline França & 7,031 & 7,082 & 7,082 & 7,082 & 19 \\
\hline Grécia & 7,082 & 7,082 & 7,082 & 7,117 & 20 \\
\hline Holanda & 7,082 & 7,082 & 7,186 & 6,457 & 23 \\
\hline Hungria & 6,444 & 6,464 & 6,464 & 7,038 & 3 \\
\hline Irlanda & 6,951 & 7,082 & 7,082 & 7,322 & 14 \\
\hline Islândia & 7,322 & 7,322 & 7,322 & 7,198 & 31 \\
\hline Itália & 7,186 & 7,186 & 7,221 & 7,273 & 27 \\
\hline Japão & 7,273 & 7,273 & 7,273 & 7,051 & 30 \\
\hline Luxemburgo & 7,003 & 7,031 & 7,118 & 6,548 & 17 \\
\hline México & 6,556 & 6,561 & 6,527 & 7,152 & 5 \\
\hline Noruega & 7,118 & 7,118 & 7,221 & 7,089 & 24 \\
\hline Nova Zelândia & 7,054 & 7,054 & 7,158 & 6,612 & 21 \\
\hline Polônia & 6,599 & 6,619 & 6,619 & 6,956 & 6 \\
\hline Portugal & 6,876 & 6,979 & 7,014 & 7,037 & 10 \\
\hline Reino Unido & 7,003 & 7,054 & 7,054 & 6,847 & 13 \\
\hline República Checa & 6,824 & 6,859 & 6,859 & 7,256 & 7 \\
\hline Suécia & 7,221 & 7,273 & 7,273 & 7,220 & 29 \\
\hline Suíça & 7,186 & 7,237 & 7,237 & 6,334 & 28 \\
\hline Turquia & 6,273 & 6,282 & 6,448 & 7,192 & 2 \\
\hline
\end{tabular}

Fonte: Elaboração dos autores. 
Quadro 1 - Países mais eficientes pertencentes ao primeiro quartil da amostra

\begin{tabular}{lcc}
\hline 2004 & 2005 & 2006 \\
\hline Brasil & Brasil & Brasil \\
Turquia & Turquia & Turquia \\
Hungria & Hungria & Hungria \\
Eslováquia & Eslováquia & México \\
México & México & Eslováquia \\
Polônia & Polônia & Polônia \\
Coréia & República Checa & República Checa \\
República Checa & Estados Unidos & Estados Unidos \\
\hline
\end{tabular}

Fonte: Elaboração dos autores.

Figure 1: Índices de ineficiência anual do Brasil e dos países da OECD

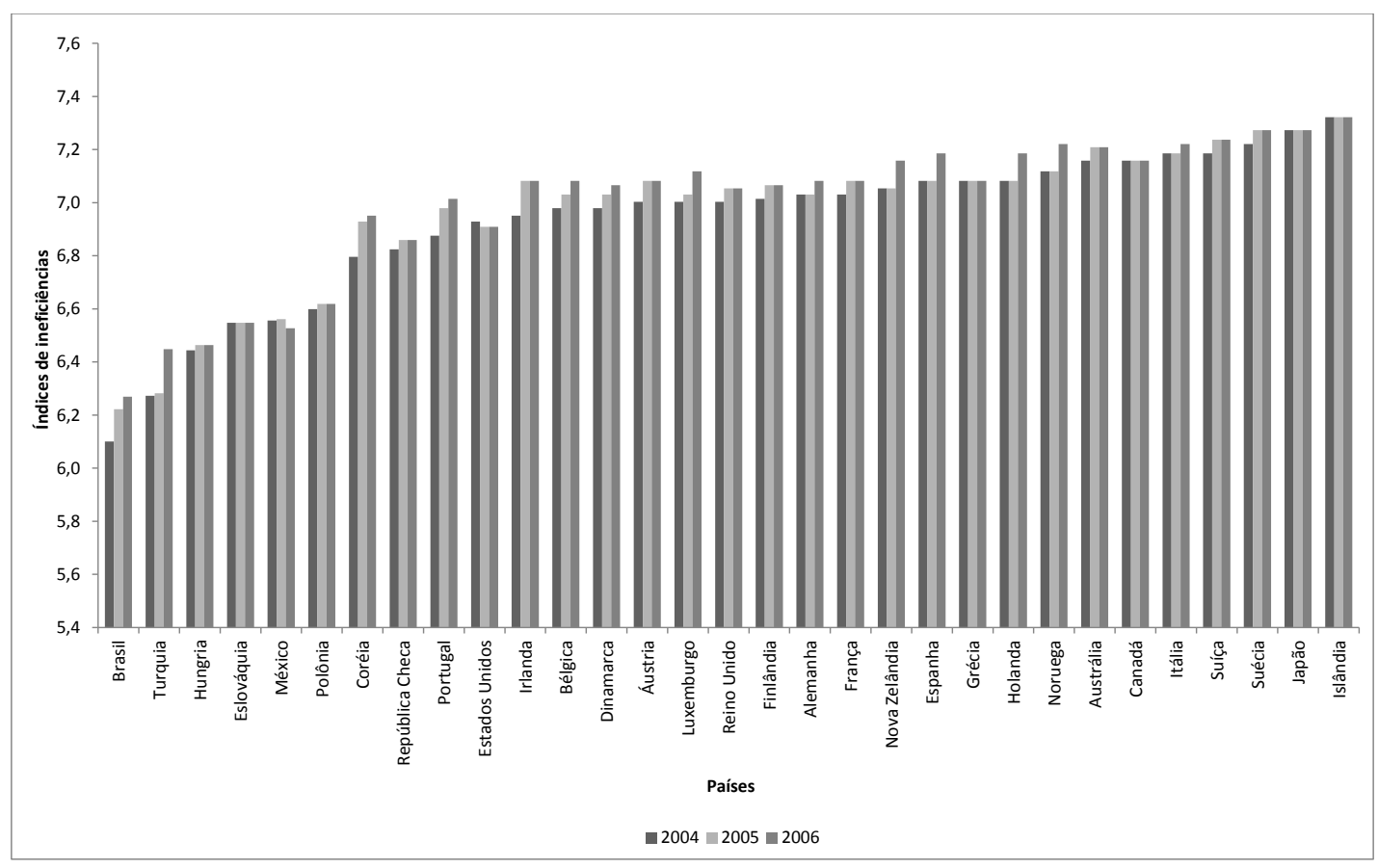

Fonte: Elaboração dos autores. 
Figure 2: Índices de ineficiências do Brasil e dos países da OECD: 2004, 2005 e 2006

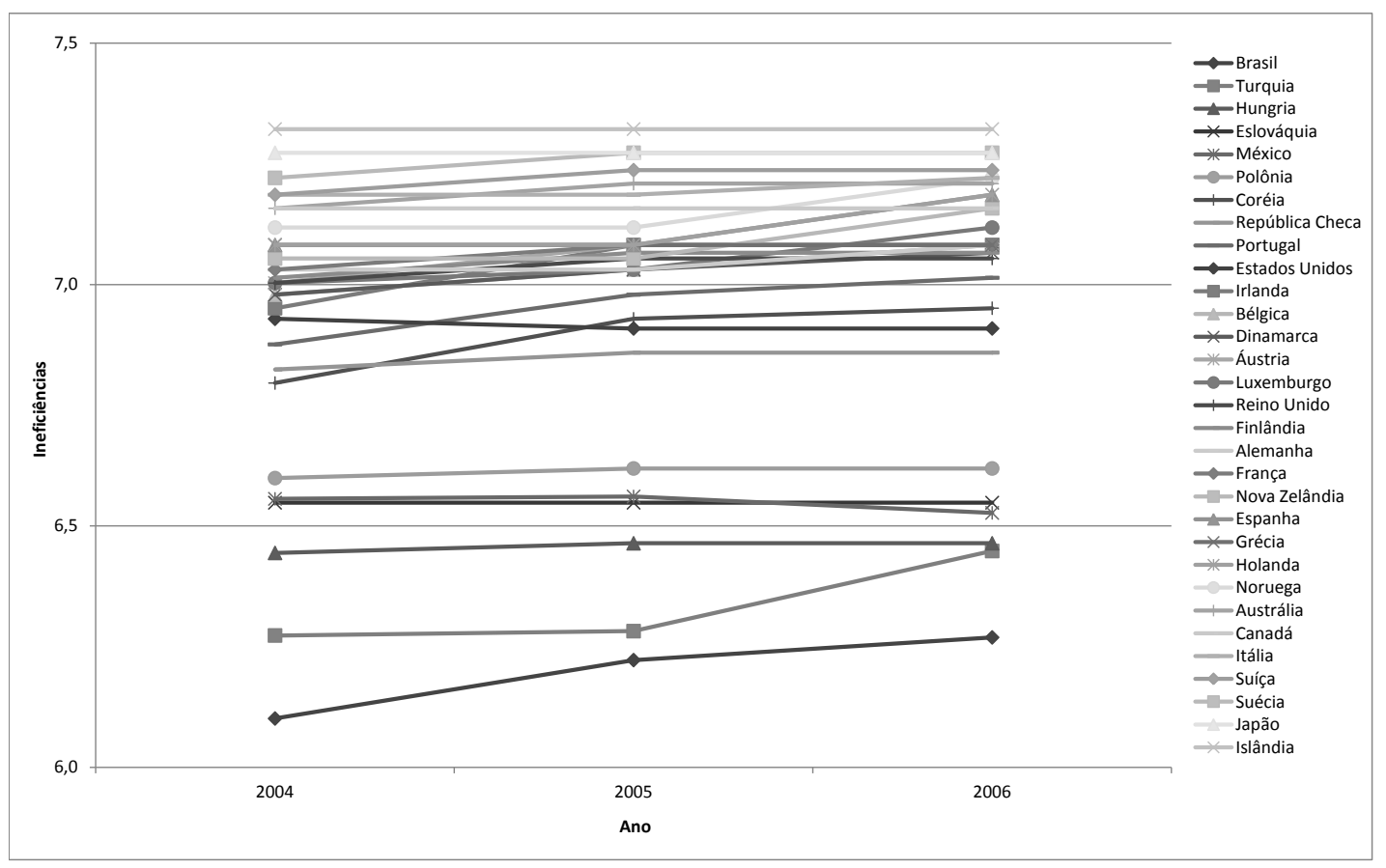

Fonte: Elaboração dos autores.

indicadores da saúde ${ }^{6}$ e, em contraponto, ainda mantém um enorme hiato com relação ao nível de gasto com saúde per capita máximo da amostra (Estados Unidos, com US\$PPP 6,714.00).

Note-se, também, que o Brasil a despeito dos indicadores de saúde desfavoráveis em relação aos países da amostra, conforme vimos na Tabela 1 tem, em termos de eficiência técnica relativa, o melhor desempenho relativo em todos os anos da análise. Como consequência, vemos, na Tabela 3 e no Quadro 1, que o Brasil seria, de fato, o primeiro colocado em um ranking de eficiência média, para o período analisado.

Uma conjectura para explicação desses resultados pode estar na sensibilidade dos gastos per capita empregados na provisão dos serviços de saúde nos indicadores de saúde que usamos. O impacto de cada unidade monetária investida em países como o Brasil, México e Turquia, que também apresentaram resultados razoavelmente satisfatórios, parece ser bem mais alta, no que tange à melhoria na saúde da população, em comparação ao observado em muitos dos países que compõem a OCDE.

Não se quer dizer aqui, de nenhum modo, que a provisão de serviços de saúde no Brasil tenha um histórico de bom desempenho, ou que este sistema apresente uma estrutura que possa ser tomada como benchmark. O que não podemos descartar, na verdade, são as amplas oportunidades de obtenção de promissoras relações de custo-efetividade em saúde no nosso país. Nosso país, a despeito dos indicadores de saúde desfavoráveis em relação aos países da amostra tem, em termos de eficiência técnica relativa, o melhor desempenho relativo em todos os anos da análise. Mas eficiência, no caso,

\footnotetext{
${ }^{6}$ Um dado que pode comprovar essa afirmação é o fato de que, em apenas um ano, a mortalidade infantil caiu 4 pontos passando de uma taxa de 32 crianças mortas em cada 1.000 nascidas no ano de 2004 para 28 no ano de 2005(http: //www. who. int/ whosis/whostat2007.pdf).
} 
significa distância em relação a um ótimo amostral. Esse conceito é diferente de efetividade, que implicaria na consecução de resultados pretendidos, que o Brasil ainda está longe de obter.

Alguns estudos classificam o Brasil em rankings de sistemas de saúde, utilizando modelos de fronteiras estocásticas de eficiência, e os resultados são os mais variados. Evans et alii (2000), utilizaram um modelo econométrico flexível (translog), aplicados em gastos com saúde, perfil educacional e utilizaram, como resultado (output), um indicador sintético de condições de saúde (o Disability Adjusted Life Expectancies - DALE). O Brasil ficou em $78^{\circ}$ lugar entre 191 países avaliados. Nesse estudo, Oman (que não faz parte da OCDE) aparece em $1^{\circ}$ lugar; Malta em $2^{\circ}$; Itália em $3^{\circ}$; França em $4^{\circ}$; Holanda em $19^{\circ}$; Bélgica em $28^{\circ}$; Turquia, $33^{\circ}$; México, $63^{\circ}$; Argentina, $71^{\circ}$; Estados Unidos em $72^{\circ}$.

Em OMS (2000), em uma amostra de 191 países, em um arcabouço de fronteiras estocásticas, o produto do sistema de saúde inclui, além da medida de saúde (o DALE), com peso de $50 \%$, um conjunto de variáveis (sinteticamente denominadas de responsiveness) relacionadas com as expectativas dos cidadãos. Nesse estudo, alguns posicionamentos interessantes seriam: Brasil em $125^{\circ}$ lugar entre 191 países avaliados, com França em $1^{\circ}$ lugar; Itália em $2^{\circ}$; Malta em $5^{\circ}$; Holanda em $17^{\circ}$; Bélgica em $21^{\circ}$; Estados Unidos em $37^{\circ}$; México, $61^{\circ}$; Turquia, $70^{\circ}$. Os países desenvolvidos, nessa análise, melhoram suas posições.

Estache et alii (2007), em um modelo econométrico de medida de eficiência, obtiveram resultados melhores para a eficiência de gastos governamentais, incluindo saúde, em países de alta renda, quando comparados com países de renda média e baixa. Mas não foi possível discriminar, com precisão, a posição isolada do sistema de saúde brasileiro no referido trabalho.

Em resumo, não parece existir consenso na literatura baseada em fronteiras estocásticas, sobre a posição relativa de serviços de saúde dos países. Acreditamos que o presente texto contribui com o debate, notadamente sob uma perspectiva voltada para a avaliação da situação brasileira, que não é foco dos demais trabalhos publicados.

\section{COMENTÁRIOS FINAIS}

O presente estudo tem como objetivo principal comparar a eficiência nos serviços de saúde do Brasil com as observadas nos países da OCDE. A despeito das usuais limitações, inclusive nos dados, recorrentes nos estudos que fazem comparações internacionais em sistemas de saúde, pudemos obter alguns achados que julgamos importantes e que resumimos a seguir.

Os resultados obtidos devem ser analisados com cuidados. É frequente que avaliações de eficiência sejam confundidas com avaliação de qualidade ou de efetividade de serviços. Embora o presente estudo envolva variáveis típicas de estudos de qualidade de sistemas de saúde, o nosso enfoque é bastante diferente desses estudos. O que procuramos investigar, por enquanto, é a relação entre os custos per capita nos sistemas de saúde analisados, e alguns indicadores selecionados no escopo de atuação das autoridades sanitárias.

Aparentemente, o desempenho relativo do sistema de saúde do Brasil face aos países da OCDE não é ruim em termos de custo-efetividade. O Brasil está longe de atingir uma estabilidade dos gastos, da estrutura, e do desempenho do seu sistema de saúde, mas tendemos a não rejeitar a hipótese de um desempenho favorável, em termos relativos, para o sistema de saúde brasileiro. Ou seja, não podemos descartar a hipótese de que o gasto per capita eficiente em saúde, no Brasil, no escopo de nosso estudo, teria retornos relativos estimados mais elevados do que os estimados para vários países desenvolvidos da OCDE. Ressalte-se, novamente, que não estamos atestando a excelência dos serviços de saúde no Brasil. Conforme comentamos, eficiência não é efetividade. Os indicadores de saúde de nosso país são ainda muito ruins, quando comparados com a média dos mesmos indicadores observados na OCDE. $\mathrm{O}$ nível de gastos per capita também é muito baixo. Em nossa interpretação, o nosso estudo revela, de modo aparentemente consistente, que existe um amplo espaço para investimentos no aprimoramento do sistema de saúde brasileiro. O Brasil é eficiente na amostra, mas não é efetivo, pois a situação 
geral de saúde da população não é comparável ao que observamos, em média, nos países da OCDE. Comparado com a amostra, o Brasil tem desempenho ruim nos indicadores de saúde, como esperança de vida ao nascer (para homens e para mulheres) e mortalidade infantil. O Brasil tem gasto per capita em saúde relativamente baixo. Mas, como a ineficiência é baixa, o desempenho do Brasil não é totalmente desfavorável pois, potencialmente, o país conseguiria transformar insumos em resultados com um aproveitamento bastante razoável.

Esse resultado não deveria ser surpreendente, pois os retornos marginais dos gastos em saúde podem ser decrescentes. ${ }^{7}$ Dito de outro modo, dependendo dos indicadores utilizados, os retornos dos gastos em saúde podem crescer cada vez menos, à medida que os gastos aumentam e que os indicadores melhoram. Assim, países com gastos mais elevados, e com melhores indicadores de saúde, podem esperar benefícios marginais menores, para cada unidade monetária adicional gasta em saúde, do que países que gastam menos e apresentam piores indicadores. Mas pode ser que isso não ocorra, o que aumentaria a distância entre os indicadores de saúde dos países desenvolvidos e os países em desenvolvimento, como o Brasil. Essa hipótese não foi especificamente testada em nosso estudo que indica apenas que, no caso brasileiro, tal distância não estaria aumentando em relação aos países da OCDE.

\section{BIBLIOGRAFIA}

Afonso, A. S. \& Aubyn, M. (2005). Non-parametric approaches to education and health expenditure efficiency in OECD countries. Journal of Applied Economics, VIII:227-246.

Aigner, D., Lovell, C. A. K., \& Schmidt, P. S. (1977). Formulation and estimation of stochastic frontier models. Journal of Econometrics, 6:21-37.

Barros, P. P. (2005). Economia da Saúde. Conceitos e Comportamento. Edições Almedina S.A., Portugal.

Battese, G. E. \& Corra, G. S. (1977). Estimation of a production frontier model: With application to the Pastoral Zone of Eastern Australia. Australian Journal of Agricultural Economics, 21:169-179.

Brasil (2006). Constituição da República Federativa do Brasil. Texto Consolidado até a Emenda Constitucional no. 52 de 08 de março de 2006. Senado Federal, Secretaria Especial de Editoração e Publicações, Brasília.

Dever, G. E. A. (1998). A Epidemiologia na Administração dos Serviços de Saúde. Pioneira, São Paulo.

Estache, A., Gonzalez, M., \& Trujillo, L. (2007). Government expenditures on education, health and infraestructure: A Naïve look at levels, outcomes and efficiency. Policy Research Working Paper 4219, World Bank.

Evans, D. B., Tandon, A., Murray, C. J. L., \& Lauer, J. A. (2000). The comparative efficiency of national health systems in producing health: An analysis of 191 countries. GPE Discussion Paper Series 29, World Health Organization.

Fried, H. O., Lovell, C. A. K., \& Schmidt, S. S. (1993). The Measurement of Productivity Efficiency. Techniques and Applications. Oxford University Press, New York.

Jacobs, R., Smith, P. c., \& Street, A. (2006). Measuring Efficiency In Health Care. Analytic Techniques and Health Policy. Cambridge University Press, Cambridge, UK.

\footnotetext{
${ }^{7}$ Ver, a esse respeito, Barros (2005), particularmente o Capítulo 4; Santerre e Neun (2000), notadamente o Capítulo 4; e Zweifel e Breyer (1997), especialmente o Capítulo 1.
} 
Maddala, K. (2001). Introduction to Econometrics. John Wiley \& Sons Ltd., England, $3^{\text {rd }}$ edition.

Meeusen, W. \& Van Den Broeck, J. (1977). Efficiency estimation from Cobb-Douglas production functions with composed error. International Economic Review, 18:435-444.

Ocké-Reis, C. O. (2006). Sistemas de Saúde Comparados: Gasto, Acesso e Desempenho. Seminários DIMAC 237. Instituto de Pesquisa Econômica Aplicada (IPEA).

OMS (2000). The World Health Report, 2000. Health Systems: Improving Performance. World Health Organization (WHO), Geneva, Switzerland.

OMS (2007). Organização Mundial da Saúde. Disponível em http://www. who.int/whosis/ whostat2007.pdf. Acessado em 14/07/2007.

Rouquayrol, M. Z. \& Almeida Filho, N. (2001). Epidemiologia e Saúde. MEDSI Editora Médica e Científica Ltda., Rio de Janeiro, 5a. edition. 1a. reimpressão.

Santerre, R. E. \& Neun, S. P. (2000). Health Economics. Theories, Insights and Industry Studies. South-Western, Ohio, USA. Revised edition.

Schmidt, P. (1988). Estimation of a fixed-effect Cobb-Douglas system using panel data. Journal of Econometrics, 37:361-80.

Souza, I. V., Nishijima, M., \& Rocha, F. (2010). Eficiência do setor hospitalar nos municípios paulistas. Economia Aplicada, 14:51-66.

Vitaliano, D. F. \& Toren, M. (1994). Cost and efficiency in nursing homes: A stochastic frontier approach. Journal of Health Economics, 13:281-300.

Zweifel, P. \& Breyer, F. (1997). Health Economics. Oxford University Press Inc., New York, USA. 


\section{A. APÊNDICE I: A CORRELAÇÃO ENTRE VARIÁVEIS}

Para simplificar os modelos, e com o intuito de evitar correlações muito fortes entre variáveis dependentes nas regressões, foi elaborada a matriz de correlação entre as variáveis explicativas dos Gastos per Capita, como foi o caso da esperança de vida ao nascer de homens e de mulheres. Uma variável explicativa-chave para o Gasto per Capita, após inúmeras tentativas, e combinações de variáveis, foi o Índice de Sobrevivência Infantil - ISR.

Table A-1: Matriz de correlação: Ano de 2004

\begin{tabular}{|c|c|c|c|c|}
\hline & $\begin{array}{l}\text { Gasto com saúde per capita } \\
\text { (USSPPP) }\end{array}$ & $\begin{array}{l}\text { Esperança de vida ao nascer } \\
\text { (homens) }\end{array}$ & $\begin{array}{l}\text { Esperança de vida ao nascer } \\
\text { (mulheres) }\end{array}$ & $\begin{array}{l}\text { Índice } \\
\text { de sobrevivência infantil }\end{array}$ \\
\hline $\begin{array}{l}\text { Gasto com saúde per capita } \\
\text { (US\$ PPP) }\end{array}$ & 1,000 & & & \\
\hline $\begin{array}{l}\text { Esperança de vida ao nascer } \\
\text { (homens) }\end{array}$ & 0,638 & 1,000 & & \\
\hline $\begin{array}{l}\text { Esperança de vida ao nascer } \\
\text { (mulheres) }\end{array}$ & 0,535 & 0,930 & 1,000 & \\
\hline $\begin{array}{l}\text { Índice } \\
\text { de sobrevivência infantil }\end{array}$ & 0,435 & 0,744 & 0,764 & 1,000 \\
\hline
\end{tabular}

Table A-2: Matriz de correlação: Ano de 2005

\begin{tabular}{llll}
\hline & $\begin{array}{l}\text { Gasto com saúde per capita } \\
\text { (USSPPP) }\end{array}$ & $\begin{array}{l}\text { Esperança de vida ao nascer } \\
\text { (homens) }\end{array}$ & $\begin{array}{l}\text { Esperança de vida ao nascer } \\
\text { (mulheres) }\end{array}$ \\
\hline $\begin{array}{l}\text { Gasto com saúde per capita } \\
\text { (US\$ PPP) }\end{array}$ & 1,000 & $\begin{array}{l}\text { Índice } \\
\text { de sobrevivência infantil }\end{array}$ \\
$\begin{array}{l}\text { Esperança de vida ao nascer } \\
\text { (homens) }\end{array}$ & 0,624 & 1,000 \\
$\begin{array}{l}\text { Esperança de vida ao nascer } \\
\text { (mulheres) }\end{array}$ & 0,528 & 0,922 & 1,000 \\
& 0,410 & 0,690 & 0,679 \\
\hline $\begin{array}{l}\text { Índice } \\
\text { de sobrevivência infantil }\end{array}$ & & \\
\hline Fonte: Elaboração dos autores. & &
\end{tabular}

Fonte: Elaboração dos autores. 
Table A-3: Matriz de correlação: Ano de 2006

\begin{tabular}{llll}
\hline & $\begin{array}{l}\text { Gasto com saúde per capita } \\
\text { (US\$PP) }\end{array}$ & $\begin{array}{l}\text { Esperança de vida ao nascer } \\
\text { (homens) }\end{array}$ & $\begin{array}{l}\text { Esperança de vida ao nascer } \\
\text { (mulheres) }\end{array}$ \\
\hline $\begin{array}{l}\text { Gasto com saúde per capita } \\
\text { (US\$ PPP) }\end{array}$ & 1,000 & $\begin{array}{l}\text { Índice } \\
\text { de sobrevivência infantil }\end{array}$ \\
$\begin{array}{l}\text { Esperança de vida ao nascer } \\
\text { (homens) }\end{array}$ & 0,629 & 1,000 \\
$\begin{array}{l}\text { Esperança de vida ao nascer } \\
\text { (mulheres) }\end{array}$ & 0,541 & 0,895 & 1,000 \\
& 0,405 & 0,646 & 0,722 \\
\hline $\begin{array}{l}\text { Índice } \\
\text { de sobrevivência infantil }\end{array}$ & & & 1,000 \\
\hline
\end{tabular}




\section{B. APÊNDICE II: A ESCOLHA DAS VARIÁVEIS}

Table B-4: Time-invariant inefficiency model

\begin{tabular}{|c|c|c|c|c|c|c|}
\hline \multirow[t]{2}{*}{ Gasto com saúde per capita (USS PPP) } & \multirow[t]{2}{*}{ Coeficiente } & \multirow[t]{2}{*}{ Desvio padrão } & \multirow[t]{2}{*}{$Z$} & \multirow[t]{2}{*}{$P>|z|$} & \multicolumn{2}{|c|}{ Intervalo de confiança } \\
\hline & & & & & Low & Up \\
\hline Intercepto & $-17,632$ & 2,801 & $-6,290$ & 0,000 & $-23,121$ & $-12,142$ \\
\hline Esperança de vida ao nascer (homens) & 5,647 & 0,654 & 8,630 & 0,000 & 4. 365 & 6,929 \\
\hline$/ \mathrm{mu}$ & 0,956 & 0,117 & 8,170 & 0,000 & 0,726 & 1,185 \\
\hline /nsigma2 & $-1,635$ & 0,306 & $-5,350$ & 0,000 & $-2,235$ & $-1,036$ \\
\hline /ilgtgamma & 4,728 & 0,367 & 12,890 & 0,000 & 4,009 & 5,447 \\
\hline sigma2 & 0,195 & 0,060 & & & 0,107 & 0,355 \\
\hline gamma & 0,991 & 0,003 & & & 0,982 & 0,996 \\
\hline sigma_u2 & 0,193 & 0,060 & & & 0,076 & 0,310 \\
\hline sigma_v2 & 0,002 & 0,000 & & & 0,001 & 0,002 \\
\hline Obs. & ll(null) & ll(model) & $\mathrm{df}$ & AIC & BIC & \\
\hline 93 & . & 75,6438 & 5 & $-141,288$ & $-128,625$ & \\
\hline
\end{tabular}

Fronteira estocástica/modelo half-normal;

Group variable (i): Países; Time variable (t): Tempo;

Number of obs $=93$; Number of groups $=31$; Obs per group: 3 ;

Wald chi2 (1) = 74,51; Log likelihood $=75,644$; Prob $>$ chi2 $=0,0000$.

Como podemos observar nas duas tabelas anteriores, a variável "esperança de vida ao nascer para homens" é mais adequada do que a "esperança devida ao nascer para mulheres" como variável dependente no modelo, pois possui o menor valor para o critério de informação de Akaike inclusivo AIC. 
Table B-5: Time-invariant inefficiency model

\begin{tabular}{|c|c|c|c|c|c|c|}
\hline \multirow[t]{2}{*}{ Gasto com saúde per capita (US\$ PPP) } & \multirow[t]{2}{*}{ Coeficiente } & \multirow[t]{2}{*}{ Desvio padrão } & \multirow[t]{2}{*}{$Z$} & \multirow[t]{2}{*}{$P>|z|$} & \multicolumn{2}{|c|}{ Intervalo de confiança } \\
\hline & & & & & Low & Up \\
\hline Intercepto & $-15,391$ & 2,968 & $-5,190$ & 0,000 & $-21,207$ & $-9,574$ \\
\hline Esperança de vida ao nascer (mulheres) & 5,051 & 0,683 & 7,390 & 0,000 & 3,712 & 6,390 \\
\hline$/ \mathrm{mu}$ & 0,880 & 0,136 & 6,490 & 0,000 & 0,615 & 1,146 \\
\hline /lnsigma2 & $-1,284$ & 0,344 & $-3,730$ & 0,000 & $-1,959$ & $-0,609$ \\
\hline /ilgtgamma & 4,878 & 0,397 & 12,300 & 0,000 & 4,100 & 5,655 \\
\hline sigma2 & 0,277 & 0,095 & & & 0,141 & 0,544 \\
\hline gamma & 0,992 & 0,003 & & & 0,984 & 0,997 \\
\hline sigma_u2 & 0,275 & 0,095 & & & 0,088 & 0,462 \\
\hline sigma_v2 & 0,002 & 0,000 & & & 0,001 & 0,003 \\
\hline Obs & ll(null) & ll(model) & df & AIC & BIC & \\
\hline 93 & . & 66,144 & 5 & $-122,289$ & $-109,626$ & \\
\hline
\end{tabular}

Fronteira estocástica/modelo half-normal;

Group variable (i): Países; Time variable (t): Tempo;

Number of obs $=93$; Number of groups $=31$; Obs per group: 3 ;

Wald chi2 $(1)=74,51 ;$ Log likelihood $=75,644 ;$ Prob $>$ chi2 $=0,0000$. 